\title{
Analysis and Comparison of Some Recent Classes of Turbo Like Codes for the Upcoming DVB Standards
}

\author{
Alexandre Graell i Amat, Guido Montorsi, and Francesca Vatta
}

\begin{abstract}
In this paper, a number of powerful recent classes of turbo like codes are analyzed as possible candidates for the upcoming DVB Standards. The final selection is justified in terms of the best tradeoff between complexity and performance. One of these classes has been recently proposed by the authors in the framework of their ongoing work on the technologies, requested by the DVB Project, which could be considered as candidates for the second generation terrestrial transmission standard DVBT2 (specification to be completed in 2009). In particular, with the aim of maintaining the maximum compatibility with the family of DVB standards, the authors are currently addressing individual technology elements for the DVB-T2 standard, such as practical low-complexity coding structures. To this end, a serial concatenated convolutional code structure has been proposed, which is expected to overcome the turbo like coding schemes currently used in the family of DVB standards.
\end{abstract}

\section{INTRODUCTION}

In the recent years, wired and wireless networks have experienced an incredibly rapid development, driven by the growth of a myriad of new applications: video streaming, music distribution, Internet, etc. This growth has imposed strong requirements in terms of data rate, reliability and network capacity, requirements that will be much more stringent in future networks. Network designers must deal with these tremendous challenges, subject to constraints of bandwidth efficiency, transmission power, flexibility and computational complexity.

During the last years, new approaches and very promising technologies emerged to respond to these demands. On one hand, channel coding techniques have become essential in all communication systems, since they can provide both reliability and bandwidth efficiency. With the invention of Turbo Codes by $\mathrm{C}$. Berrou et al. and the emergence of turbo-like codes in

This work has been supported by Galileo 2007/2008 Project of scientific cooperation between Italy and France and by the Marie Curie Intra-European Fellowship within the 6th European Community Framework Programme. It was presented in part at the the 2007 International Conference on Software, Telecommunications and Computer Networks - SOFTCOM '07, Special Session SS1 - Digital Video Broadcasting, Split - Dubrovnik, Croatia, September 27-29, 2007.

A. Graell i Amat is with IT/Telecom Bretagne, Electronics Department, CS 83818 - 29238 Brest Cedex 3, France (e-mail: alexandre.graell@telecombretagne.eu).

G. Montorsi is with Dipartimento di Elettronica, Politecnico di Torino, Corso Duca degli Abruzzi 24, I-10129 Torino, Italy (e-mail: montorsi@polito.it).

F. Vatta is with DEEI, University of Trieste, Via A. Valerio 10, I-34127 Trieste, Italy (e-mail: vatta@units.it). general, error control coding has now reached the fundamental limits on transmission rate for the Gaussian Channel. However, the minimum Hamming distance (MHD) of a turbo code may not be sufficient to prevent a detrimental change in the error rate vs. signal to noise ratio curve, the so-called error floor.

Turbo codes (TCs) are today mainly used in Automatic ReQuest (ARQ) systems, which do not usually require very low error rates. Targeted Frame Error Rates (FER) from $10^{-2}$ to $10^{-5}$ are typical for this kind of communication systems. However, in future system generations, lower error rates, down to $10^{-8}$, may be necessary to open the way to real-time and more demanding applications, such as Digital Video Broadcasting or videoconferencing. This calls for more powerful coding schemes, capable of achieving very low error rates, yet maintaining a reasonable complexity, and for wideband wireless and mobile communication systems enabling multimedia services in extraordinary hostile propagation environments where the limited availability of the radio frequency band is a major obstacle. The most promising approach to achieve this aim is to employ multiple antennas at both the transmitter side and the receiver side together with multicarrier modulation (OFDM or MC-CDMA) for ad-hoc and cellular network settings.

With this in mind, it is evident that future market pressures will be for lower and more flexible bit rates, better spectrum and power efficiency, and more robust reception, through a MIMO technology using a flexible number of multiple antennas at both the transmitter side and the receiver side, since this technology promises enormous data rate increases (multiplexing gain) as well as increased robustness against fading (diversity gain). Therefore, constructing practical lowcomplexity coding structures that achieve these gains and perform close to optimal becomes a relevant and challenging problem. In this environment, concatenated codes are expected to perform close to the fundamental limits of the channel and to overcome the principal disadvantages of other coding schemes currently used in the family of DVB standards.

In this paper, we provide a performance analysis and a comparison of a number of powerful classes of turbo like codes, proposed recently, which could be considered as candidates for the upcoming DVB standards. One of these classes, based on a variable rate serial concatenated convolutional code structure, has been recently proposed by the authors in the framework of their ongoing work on the technologies, requested by the DVB Project, which could be considered as candidates for 
the second generation terrestrial transmission standard DVBT2 (specification to be completed in 2009). In particular, the authors are currently addressing individual technology elements for the DVB-T2 standard, such as practical lowcomplexity coding structures. To this end, a serial concatenated convolutional code (SCCC) structure has been proposed in [1, 2] and [3].

The objective of this paper is to validate the possible candidacy of this new class of SCCCs to be used in the DVBT2 standard. To this end, a comparison of its performances with the performances of other newly proposed turbo-like codes is needed, taking into account not only the obtainable residual Frame Error Rate (FER) at the decoder output but also the co-decoder complexity. In particular, the comparison will be performed with respect to some well performing parallel concatenated codes structures, such as the well-known Doublebinary Circular Recursive Convolutional (CRSC) code, which is currently used in the DVB-RCS standard, and with respect to other competitive serial concatenated code structures proposed recently.

The paper is organized as follows. In Section II we present the requirements of the upcoming DVB-T2 standard. In Section III we recall the properties of a new class of Turbo Like Codes presented recently in [1-3]. In Section IV we compare its performance, through union bounds and simulation results, with those of other parallel concatenated codes structures, such as the CRSC codes, and other competitive recently proposed serial concatenated code structures. Finally, in Section IV we summarize the main conclusions.

\section{THE UPCOMING DVB-T2 STANDARD}

DVB-T has established itself as the leading specification for digital terrestrial TV broadcasting. Since DVB-T was initially specified, there have been substantial developments in both the modulation technology available and the economics of the transmission chain. There are new ways of modulating and error-protecting the broadcast stream, the commercial value of digital broadcast spectrum is on a rising trend and the cost and feasibility of including much more sophisticated technology at an acceptable cost in receivers has enabled a different balance to be struck between receiver complexity and bandwidth efficiency. We can also anticipate greater convergence between traditional broadcast services and telecommunications services, including mobile and broadband services. All these trends together with the increased capacity needs of High Definition TV (HDTV) give rise to the need to specify a second generation enhanced terrestrial DVB. It is an open question whether the future TV applications require an ability to view the same signals on different devices with varying screen resolutions (e.g., mobile and fixed receivers), or the use of simultaneous distribution of the same service in different formats to suit each community of receivers and users. The enhanced DVB specification would therefore need to be capable of supporting both of these scenarios. The switch off of analogue TV across the EU Broadcast Region will release the legacy analogue TV Spectrum for new applications and services. This transition will create a window of opportunity for the introduction of new technologies and the short term requirement for a DVB-T2 Specification is to be ready for deployment on the new frequency allocations defined by the Geneva 2006 Agreement (GE06) post analogue TV switch off. In addition to being ready in time for analogue $\mathrm{TV}$ switch off, a new DVB-T2 Specification must offer significant benefits in terms of payload capacity over the existing DVB-T Specification in order to be commercially viable.

It is anticipated that future DVB-T2 broadcasts will have to co-exist with existing DVB-T broadcasts for a long period of time. The DVB-T and DVB-T2 systems shall be able to be operated simultaneously without mutual interference. High spectrum efficiency together with reasonable network costs is of fundamental importance using DVB-T2, either alone or together with DVB-T. In order to allow the new DVBT2 standard to be used globally it is important to include a large flexibility with regard to frequency bands and channel bandwidths. The system is required to include a large degree of flexibility in terms of combinations of throughput and robustness, so that the operator can freely choose the desirable combination. In addition the system shall be designed in such a way that it provides an as large as possible immunity (subject to cost and other constraints) against impulsive noise interference, time variations of the wanted DVB-T2 signal, as well as against unwanted interfering transmissions. In particular:

1) The DVB-T2 specification shall offer a choice of various robustness and protection levels to be applied equally on all data of a transport stream carried by a DVB-T2 signal in a particular channel.

2) The DVB-T2 specification should offer a choice of various robustness and protection levels for each service separately, within a transport stream carried by a DVBT2 signal in a particular channel. When more than one transport stream is carried by a DVB-T2 signal in a particular channel the DVB-T2 specification should offer a choice of various robustness and protection levels for each transport stream separately.

3) The DVB-T2 specification shall provide a quality of service across the whole channel that approximates to no more than one corrupted event (to any audio, video or data services) per hour for HDTV and SDTV services.

\section{A New Class of Turbo Like Codes for the UPCOMING DVB STANDARDS}

A powerful class of rate-compatible SCCCs with low complexity has been proposed in [1,2] and [3]. Afterwards, it has been proposed as candidate for the second generation terrestrial transmission standard DVB-T2 (specification to be completed in 2009), on the basis of its capability to fulfill the requirements of variable robustness and protection levels included in the DVB-T2 specification.

The proposal of adoption of rate-compatible SCCCs, proposed in [1,2], in this environment is justified by the fact that these codes are expected to perform close to the fundamental limits of the channel and to overcome the principal disadvantages of other coding schemes, e.g., of LDPC (Low 
Density Parity Check) Codes, currently used in the DVB-S2 standard, whose performance decays rapidly if strict latency requirements are to be fulfilled, as for real time services. To this end, in [4] Parallel concatenated convolutional codes (PCCCs), Serial concatenated convolutional codes (SCCCs), and LDPC codes have been compared in terms of their performance, complexity, flexibility, and maturity. It turned out that, for the short block size, the PCCC scheme offers the best performance. It yields a gain from $0.3 \mathrm{~dB}$ (rate 1/3) to $1.05 \mathrm{~dB}$ (rate 9/10) over SCCC, and from $0.5 \mathrm{~dB}$ (rate 1/3) to $1 \mathrm{~dB}$ (rate 9/10) over LDPC. For the long block size, the LDPC scheme offers the best performance but is close to those of the SCCC and PCCC (gains from 0.15 to $0.25 \mathrm{~dB}$ ), except for PCCC rate 9/10, which has greater losses for SNR greater than $4.3 \mathrm{~dB}$. As for complexity, the SCCC scheme is simplest in terms of RAM occupation for all block sizes and rates. On the other hand, the PCCC scheme only requires a small amount of ROM to store the permutation parameters. LDPC is by far the most complex, due to the fact that the number of edges is much larger than the interleaver size of SCCC and PCCC. As for the arithmetic complexity, the SCCC scheme is by far the simplest. It is roughly two to three times less complex than the PCCC. The LDPC schemes are from two to more than six times more complex (depending on the specific case considered); this stems from the larger number of edges and required iterations for LDPC schemes, which is not compensated for by the lower complexity of check node operation with respect to the single-input single-output (SISO) processor. As for flexibility, this was evaluated referring to the possibility (and related complexity/performance consequences) of the co-decoder to adapt to different code rates, modulation schemes, and block sizes on a per frame basis. All schemes can adapt easily to variations in the modulation schemes as they are pragmatic schemes (i.e., all make use of a binary encoder whose output bits are mapped to all modulation schemes). As for code rate adaptation, the SCCC scheme adapts very easily to different code rates. The same is true for the PCCC scheme. On the other hand, adaptation to different code rates requires in general that the LDPC codes work with different codes, which have to be stored, even if several techniques have been proposed recently to design LDPC codes that achieve good rate granularity [5,6]. As for maturity, PCCCs have been already implemented in numerous cases, and accepted as standards in several system applications. SCCCs have seen fewer implementations, but their technology should not pose any further problems. LDPC codes are relatively newer in terms of applications, although their acceptance in the new DVB-S2 standard should accelerate full comprehension and solution of implementation problems.

From the considerations above, it turns out that it is worth supporting this class of SCCCs as a candidate. However, a comparison of its performances with the performances of other turbo-like codes is needed, taking into account not only the obtainable residual Frame Error Rate (FER) at the decoder output but also the co-decoder complexity.

In contrast to classical SCCCs, characterized by the concatenation of an outer code with a rate $R_{I}<1$ inner code, the codes proposed in [1-3] allow the inner code to be punctured

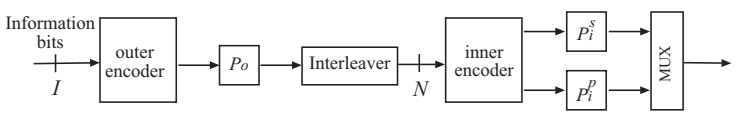

Fig. 1. Block diagram of the Serial concatenated code scheme.

beyond the unitary rate. These codes achieve a high level of flexibility and offer good performance over a wide range of code rates, using simple constituent codes.

The key idea in [1-3] is to replace the puncturing of the outer code with the puncturing of the inner code. Since in this way the rate of the outer code is fixed, this approach leads to a constant outer-code block length, thus maintaining the outer-code distance spectrum properties. As a result, a constant interleaver gain is obtained for all code rates, significantly lowering the error floor at all rates. The improvements are particularly substantial for high rate codes.

Design criteria for this class of SCCCs were suggested in $[1,2]$ based on union bounds (UBs) for the error probability [7]. This new class of codes is obtained through the serial concatenation of two systematic recursive convolutional encoders. To obtain high rates both encoders are punctured. However, in contrast to standard SCCCs where high rates are obtained by concatenating an extensively punctured outer encoder with an inner encoder of rate $R_{c}^{i} \leqslant 1$ such that the rate of the SCCC, $R_{\mathrm{SCCC}}$, is at most equal to the rate of the outer encoder $R_{c}^{o}$ $\left(R_{\mathrm{SCCC}} \leqslant R_{c}^{o}\right)$, the inner encoder in Fig. 1 can be punctured beyond the unitary rate, i.e., the overall code rate $R_{\mathrm{SCCC}}$ can be greater than the outer code rate $R_{c}^{o}$. Moreover, as made evident in the figure, puncturing is not directly applied to the inner code sequence but split into two different puncturings, in correspondence to inner code systematic bits and inner code parity bits $\left(P_{i}^{s}\right.$ and $P_{i}^{p}$, respectively). Assuming an inner mother code of rate $1 / n$, the rate of the resulting SCCC is given by

$$
R_{\mathrm{SCCC}}=R_{c}^{o^{\prime}} R_{c}^{i}=R_{c}^{o^{\prime}} /\left(\rho_{s}+(n-1) \rho_{p}\right),
$$

where $R_{c}^{o^{\prime}}$ is the outer code rate after applying the fixed puncturing pattern $P_{o}$, and $\rho_{s}\left(\rho_{p}\right)$ is the systematic permeability (parity permeability) rate, defined as the proportion of inner code systematic bits (parity bits) which are not punctured [8].

This particular code structure offers superior performance to that of standard SCCC, especially for high-rates. Notice that for high rates, the exhaustive puncturing of the outer code leads to a poor code in terms of free distance, thus leading to a higher error floor. On the contrary, the code structure discussed here, keeps the interleaver gain for low rates also in the case of very high rates, since the heavy puncturing is moved to the inner encoder. Moreover it is well suited for rate-compatible schemes.

The code optimization criteria proposed in $[1,2]$ targeted the error floor (EF) region, leading to correspondingly good code constructions for this region. In particular, in $[1,2]$ we have drawn the following design considerations.

- As for traditional SCCC, $P_{o}$ should be chosen to optimize the outer code output distance spectrum. 
- For a fixed pair $\rho_{s}$ and $\rho_{p}$, the pattern $P_{i}^{p}$ must be optimized to yield the best inner encoder Input-Output Weight Enumerating Function (IOWEF). Furthermore, the deinterleaved version of the puncturing pattern $P_{i}^{s}$, $P^{\prime}\left(P^{\prime}=\pi^{-1}\left[P_{i}^{s}\right]\right)$ must be optimized to yield the best outer encoder Output Weight Enumerating Function (OWEF). Notice that this is equivalent to optimize the outer code $\mathcal{C}_{o}$, punctured through $P_{o}$ and a pattern $P^{\prime}$ with permeability $\rho_{s}$, and then set $P_{i}^{s}$ to be equal to the interleaved version of $P^{\prime}$, i.e., $P_{i}^{s}=\pi\left[P^{\prime}\right]$. Therefore, $P_{i}^{s}$ turns out to be interleaver dependent.

This design approach leads to an optimal code performance in the EF region, verified in [2] estimating the code performance sensitivity to the adopted puncturing strategy, as done, e.g., in [9] for parallel concatenated codes. In essence, in $[2,9]$ it was shown that the best puncturing criterion is the one that follows the rules assessed for the design of optimum constituent codes in the same concatenation scheme. For parallel concatenations, this criterion can be found in [10]. For serial concatenations, see [11]. This approach, however, provides little insight into the code convergence behavior in the waterfall (WF) region. Thus, in [3] the convergence properties of the SCCC proposed in $[1,2]$ were analyzed using extrinsic information transfer (EXIT) chart techniques.

It is clear that the performance of the SCCC code depends on puncturing patterns $P_{o}, P_{i}^{s}$ and $P_{i}^{p}$, and, subsequently, on the permeability rates $\rho_{s}$ and $\rho_{p}$, which should be properly optimized. In most cases, the optimal design criteria for the WF and EF regions do not coincide. In fact, optimizing the performance in the WF region leads to the worst possible performance in the $\mathrm{EF}$ region and viceversa for low code rates. In order to provide a suitable trade-off, a joint approach is required. Combining EXIT charts with UB techniques a family of low-complexity SCCCs with good performance in both EF and WF regions can be constructed. In [3], the key idea was to first optimize the puncturing patterns $P_{o}, P_{i}^{s}$ and $P_{i}^{p}$ based on bounding techniques, thus ensuring a good behavior in the EF. Note that using bounding techniques the rate-compatible puncturings in $P_{o}, P_{i}^{s}$ and $P_{i}^{p}$ are optimized separately, i.e., the order in which bits should be punctured within these puncturers are found independently (as in [1,2]). Based on these patterns, EXIT charts have then been applied to find the permeability rates $\rho_{s}$ and $\rho_{p}$, minimizing the convergence thresholds. In this way, a family of rate-compatible SCCCs can be obtained, that offer good performance in both the EF and the WF regions, thus being very competitive w.r.t. other turbo like code structures.

\section{PERformanCE COMPARISON}

In order to sustain this new class of SCCCs as a possible candidate for the upcoming DVB-T2 standard, its performance and complexity have been evaluated, through union bounds and simulation results, in comparison with:

1) standard SCCCs [11];

2) standard PCCCs, such as the UMTS Turbo Code [12, 13];
3) the Double-binary Circular Recursive Convolutional (CRSC) code presented in [14], which is currently used in the DVB-RCS standard [15];

4) a new class of serial concatenated codes introduced in [16] and called "FlexiCode". This class has been selected for comparison since it presents some similarities with the new class of SCCCs described in [1-3].

\section{A. Union Bound to the Error Probability}

Following the derivations in [17] and [11] for PCCC and SCCC, in $[1,2]$ we have derived the union bound on the bit error probability and on the frame error probability for the code construction of Fig. 1. In this subsection we recall these bounds for consistency sake.

The bit error probability of a SCCC can be upper bounded through $[1,2]$

$$
\begin{aligned}
& P_{b}(e)<\left.\sum_{w=w_{m}^{o}}^{N R_{c}^{o^{\prime}}} \frac{w}{N R_{c}^{o^{\prime}}} A^{C_{\mathrm{SCCC}}}(w, H)\right|_{H=e^{-\frac{R_{\mathrm{SCCC}} E_{b}}{N_{0}}}} \\
& =\sum_{h=h_{m}}^{N / R_{c}^{i}} \sum_{w=w_{m}^{o}}^{N R_{c}^{o^{\prime}}} \frac{w}{N R_{c}^{o^{\prime}}} A_{w, h}^{C_{\mathrm{SCCC}}} e^{-\frac{h R_{\mathrm{SCCC} E_{b}}}{N_{0}}}
\end{aligned}
$$

where $w_{m}^{o}$ is the minimum weight of an input sequence generating an error event of the outer code, $N$ is the interleaver length, $h_{m}$ is the minimum weight of codewords of the SCCC, $C_{\mathrm{SCCC}}$, of rate $R_{\mathrm{SCCC}}$, and $A^{C_{\mathrm{SCCC}}}(w, H)$ is the Conditional Weight Enumerating Function (CWEF) of the overall SCCC code.

Equivalently, the frame error probability of a SCCC can be upper bounded through

$$
\begin{aligned}
P_{f}(e) & <\left.\sum_{w=w_{m}^{o}}^{N R_{c}^{o^{\prime}}} A^{C_{\mathrm{SCCC}}}(w, H)\right|_{H=e^{-\frac{R_{\mathrm{SCCC}} E_{b}}{N_{0}}}} \\
& =\sum_{h=h_{m}}^{N / R_{c}^{i}} \sum_{w=w_{m}^{o}}^{N R_{c}^{o^{\prime}}} A_{w, h}^{C_{\mathrm{SCCC}}} e^{-\frac{h R_{\mathrm{SCCC} E_{b}}}{N_{0}}}
\end{aligned}
$$

For a generic serially concatenated code, consisting of the serial concatenation of an outer code $C_{o}$ with an inner code $C_{i}$ through a uniform interleaver, the CWEF of the overall SCCC code $A_{w, h}^{C_{\mathrm{SCCC}}}$ can be calculated exploiting the properties of the uniform interleaver, which transforms a codeword of weight $l$ at the output of the outer encoder into all distinct $\left(\begin{array}{c}N \\ l\end{array}\right)$ permutations. As a consequence, each codeword of the outer code $C_{o}$ of weight $l$, through the action of the uniform interleaver, enters the inner encoder generating $\left(\begin{array}{c}N \\ l\end{array}\right)$ codewords of the inner code $C_{i}$. The CWEF of the overall SCCC code $A_{w, h}^{C_{\mathrm{SCC}}}$ is given by $[1,2]$

$$
A_{w, h}^{C_{\mathrm{SCCC}}}=\sum_{l=0}^{N} \frac{A_{w, l}^{C_{o}} \times A_{l, h}^{C_{i}}}{\left(\begin{array}{c}
N \\
l
\end{array}\right)}
$$

where $A_{w, l}^{C_{o}}$ and $A_{l, h}^{C_{i}}$ are the CWEFs of the outer and inner codes, respectively. 


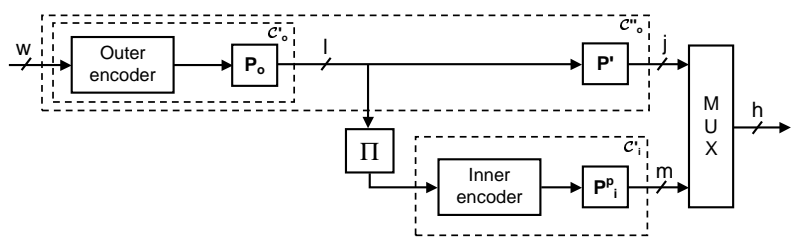

Fig. 2. Modified block diagram of the serial concatenated scheme.

This is basically the same result obtained in [11]. However, and this is the key novelty of our analysis, to evaluate the performance of the code structure of Fig. 1, instead of proceeding, as in [11], using (4), it is more suitable to refer to Fig. 2, which properly redraws the encoder of Fig. 1, for the derivation of the upper bound. Fig. 2 allows us to decouple the contributions of the inner code puncturings $P_{i}^{s}$ and $P_{i}^{p}$ to the error probability bound. Call $C_{o}^{\prime \prime}$ the code obtained from the puncturing of the outer code $C_{o}$ through $P_{o}$ and $P^{\prime}$ (the deinterleaved version of $\left.P_{i}^{s}, P^{\prime}=\pi^{-1}\left[P_{i}^{s}\right]\right), C_{o}^{\prime}$ the code obtained from the puncturing of the outer code $C_{o}$ through $P_{o}$, and $C_{i}^{\prime}$ the inner encoder $C_{i}$ generating only parity bits punctured through $P_{i}^{p}$, which is fed with an interleaved version of codewords generated by $C_{o}^{\prime} 1$. Now, the serial concatenated code structure under consideration can be interpreted as the parallel concatenation of the code $C_{o}^{\prime \prime}$ and $C_{i}^{\prime}$. Therefore, the SCCC codeword weight $h$ can be split into two contributions $j$ and $m$, corresponding to the output weights of the codewords generated by encoder $C_{o}^{\prime \prime}$ and the inner encoder $C_{i}^{\prime}$, respectively, such that $h=j+m$. With reference to Fig. 2, equation (4) can then be rewritten as $[1,2]$

$$
A_{w, h}^{C_{\mathrm{SCCC}}}=A_{w, j+m}^{C_{\mathrm{SCCC}}}=\left.\sum_{l=d_{\mathrm{f}}^{o^{\prime}}}^{N} \sum_{j=d_{\mathrm{f}}^{o^{\prime \prime}}}^{N / R_{c}^{o^{\prime \prime}}} \frac{A_{w, l, j}^{C_{o}^{\prime \prime}} \times A_{l, m}^{C_{i}^{\prime}}}{\left(\begin{array}{c}
N \\
l
\end{array}\right)}\right|_{j+m=h}
$$

where $d_{\mathrm{f}}^{o^{\prime}}$ is the free distance of the code $C_{o}^{\prime}$ and $d_{\mathrm{f}}^{o^{\prime \prime}}$ is the free distance of the code $C_{o}^{\prime \prime}$. In (5), $R_{c}^{o^{\prime \prime}}$ is the rate of the code $C_{o}^{\prime \prime}, A_{w, l, j}^{C_{o}^{\prime \prime}}$ indicates the number of codewords of $C_{o}^{\prime \prime}$ of weight $j$ associated with a codeword of $C_{o}^{\prime}$ of weight $l$ generated from an information word of weight $w$, and $A_{l, m}^{C_{i}^{\prime}}$ indicates the number of codewords of $C_{i}^{\prime}$ of weight $m$ associated with a codeword of $C_{o}^{\prime}$ of weight $l$.

The derivation of the union bound on the Error Probability for the standard SCCCs [11], the standard PCCCs, such as the UMTS Turbo Code [12,13], and the Double-binary Circular Recursive Convolutional (CRSC) code, presented in [14], are well known. As far as the new class of serial concatenated codes introduced in [16] and called "FlexiCode" is concerned, the derivation of the union bound on the Error Probability is shown in [16] and is rewritten here using the notation of Eq. (2) for consistency sake.

\footnotetext{
${ }^{1}$ Notice that, in abuse of notation, we have maintained the terminology outer encoder and inner encoder in Fig. 2 though they do not strictly act as outer and inner encoders. However, we believe that this notation reflects better the correspondence with Fig. 1 .
}

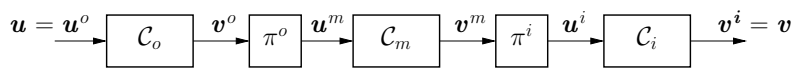

Fig. 3. Encoder structure for a generic double serially concatenated code.

The structure of the "FlexiCode" encoder is shown in [16]. The outer code is a rate-1/3 systematic feedforward convolutional code with generator matrix $G(D)=\{1,1+$ $\left.D^{2}, 1+D+D^{2}\right\}$. The inner code is a SPC code followed by a rate-1 recursive convolutional code with generator matrix $G(D)=\left\{\frac{1+D^{2}}{1+D+D^{2}}\right\}$ or $G(D)=\left\{\frac{1+D}{1+D+D^{2}}\right\}$, respectively. The first $G(D)$ matrix describing the rate-1 inner recursive convolutional code is used for an overall rate of $1 / 2$ only. The key idea is the use of a direct concatenation of the SPC with the recursive inner convolutional code to adjust the rate.

The uniform interleaver analysis applied to the "FlexiCode" described in [16] can be conducted by treating the whole coding structure as a double-serially concatenated code (DSCC) whose generic structure is shown in Fig. 3. The outer encoder $C_{o}$ has a rate $R^{o}$ and generates the code sequence $v^{o}$ from the information sequence $u=u^{o}$. The outer permutation $\pi^{o}$ then maps $v^{o}$ to the sequence $u^{m}$, which is fed to the middle recursive convolutional code $C_{m}$ of rate $R^{m}$. The resulting code sequence $v^{m}$ is permuted by the inner permutation $\pi^{i}$, which provides the input sequence $u^{i}$ for the inner rate $R^{i}$ recursive convolutional code $C_{i}$. The resulting code sequence is denoted by $v=v^{i}$.

We follow the approach of Benedetto and Montorsi [11], [18] for serially concatenated and double serially concatenated codes. The component codes of the double-serially concatenated codes are connected through uniform random interleavers. The important property of the uniform interleaver is that its output only depends on the input weight $w$, not on the distribution of the weight within the input word. A uniform interleaver of length $N^{\prime}$ maps an input weight of $w$ into all of its $\left(\begin{array}{c}N^{\prime} \\ w\end{array}\right)$ possible permutations with equal probability. As a consequence, each codeword of the outer code $C_{o}$ of weight $l_{1}$, through the action of the uniform interleaver, enters the middle encoder generating $\left(\begin{array}{c}N_{1} \\ l_{1}\end{array}\right)$ codewords of the inner code $C_{m}$, and each codeword of the middle code $C_{m}$ of weight $l_{2}$, through the action of the uniform interleaver, enters the inner encoder generating $\left(\begin{array}{c}N_{2} \\ l_{2}\end{array}\right)$ codewords of the inner code $C_{i}$. The resulting Input-Output Weight Enumerating Function (IOWEF) $\bar{A}_{w, h}^{C_{\mathrm{DSCC}}}$ is the average number of codewords in the overall DSCC $C_{\mathrm{DSCC}}$ of weight $h$ associated with an input word of weight $w$ over the ensemble of all interleavers and is given by

$$
\bar{A}_{w, h}^{C_{\mathrm{DSCC}}}=\sum_{l_{1}=0}^{N_{1}} \sum_{l_{2}=0}^{N_{2}} \frac{A_{w, l_{1}}^{C_{o}} A_{l_{1}, l_{2}}^{C_{m}} A_{l_{2}, h}^{C_{i}}}{\left(\begin{array}{c}
N_{1} \\
l_{1}
\end{array}\right)\left(\begin{array}{c}
N_{2} \\
l_{2}
\end{array}\right)}
$$

where $A_{w, l_{1}}^{C_{o}}, A_{l_{1}, l_{2}}^{C_{m}}$ and $A_{l_{2}, h}^{C_{i}}$ are the IOWEFs of the outer, middle, and inner codes, respectively. From the average IOWEF we can derive the average Weight Enumerator Func- 
tion (WEF) $\bar{A}^{C_{\mathrm{DSCC}}}(h)$ as

$$
\bar{A}_{h}^{C_{\mathrm{DSCC}}}=\sum_{w=1}^{K} \bar{A}_{w, h}^{C_{\mathrm{DSCC}}} W^{w}
$$

where $K$ is the input block length.

The average IOWEF can now be used to upper bound the error probability of the DSCC by employing the union bound. For an $(N, K)$ DSCC the bit error probability on an additive white Gaussian noise (AWGN) channel with bit SNR equal to $E_{b} / N_{0}$ can, for maximum likelihood decoding, be upper bounded by

$$
P_{b}(e)<\sum_{h=1}^{N} \sum_{w=1}^{K} \frac{w}{K} \bar{A}_{w, h}^{C_{\mathrm{DSCC}}} e^{-\frac{h K E_{b}}{N N_{0}}}
$$

Equivalently, its frame error probability can be upper bounded through

$$
P_{f}(e)<\sum_{h=1}^{N} \sum_{w=1}^{K} \bar{A}_{w, h}^{C_{\mathrm{DSCC}}} e^{-\frac{h K E_{b}}{N N_{0}}} .
$$

As for the encoder structure described in [16], Eq. (6) can be rewritten as:

$$
\bar{A}_{w, h}^{C_{\text {Flexi }}}=\sum_{l=0}^{N} \frac{A_{w, l}^{C_{o}} A_{l, h}^{\text {direct }}}{\left(\begin{array}{c}
N \\
l
\end{array}\right)}
$$

being $A_{l, h}^{\text {direct }}$ in Eq. (10) given by

$$
\bar{A}_{l, h}^{\mathrm{direct}}=\sum_{s=0}^{l} \frac{A_{l, s}^{\mathrm{SPC}} A_{s, h}^{C_{i}}}{\left(\begin{array}{c}
N / J \\
s
\end{array}\right)}
$$

and being $A_{w, l}^{C_{o}}, A_{l, s}^{\mathrm{SPC}}$ and $A_{s, h}^{C_{i}}$ the IOWEFs for the outer convolutional code (CC), the single parity check (SPC) mapper and the inner $\mathrm{CC}$, respectively. In this sense, as far as the uniform interleaver analysis is concerned, the "FlexiCode" described in [16] can be treated as a double-serially concatenated code (DSCC) where the outer CC, the SPC mapper, and the inner CC are the outer, middle, and inner codes, respectively.

Substituting Eq. (11) in Eq. (10), we obtain:

$$
\bar{A}_{w, h}^{C_{\text {Flexi }}}=\sum_{l=0}^{N} \sum_{s=0}^{l} \frac{A_{w, l}^{C_{o}} A_{l, s}^{\mathrm{SPC}} A_{s, h}^{C_{i}}}{\left(\begin{array}{c}
N / J \\
s
\end{array}\right)\left(\begin{array}{c}
N \\
l
\end{array}\right)}
$$

Notice that the second sum over $s$ in Eq. (12) goes from 0 to $l$, being $s$ the output weight of the SPC (over all SPC blocks), and $l$ the output weight of the outer convolutional code. This happens because the SPC causes a reduction in weight $\delta=l-s$ when pairs of input ones fall into a parity check for the same output bit. For this reason $\delta$ can only take even values.

In [16] an upper bound to $p_{\mathrm{SPC}}(s \mid l)$ is given, where $p_{\mathrm{SPC}}(s \mid l)$ is defined as the probability of the output weight of the SPC

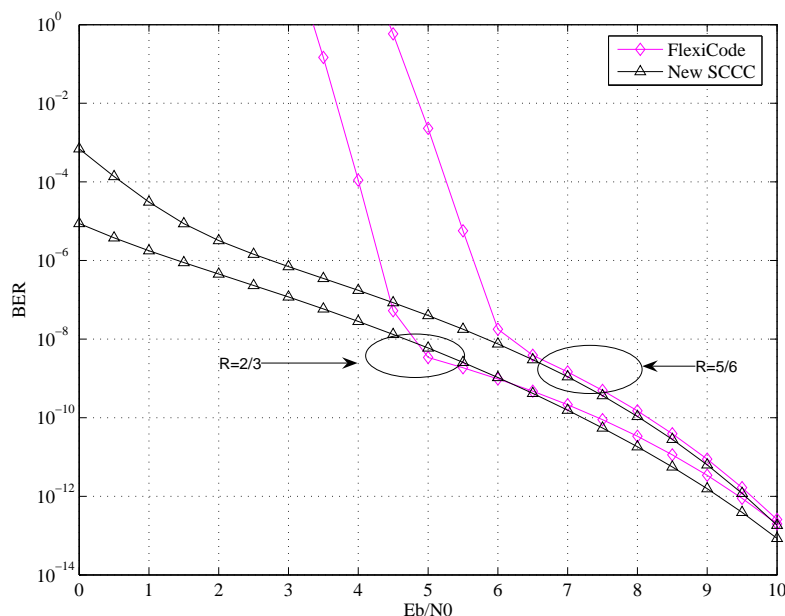

Fig. 4. Upper bounds on $P_{b}(e)$ for the new class of SCCCs (Eq. (2)) and for the "FlexiCode" (Eq. (8)) with $R=2 / 3$ and $R=5 / 6$ with $K=1504$ An AWGN channel is assumed with BPSK modulation.

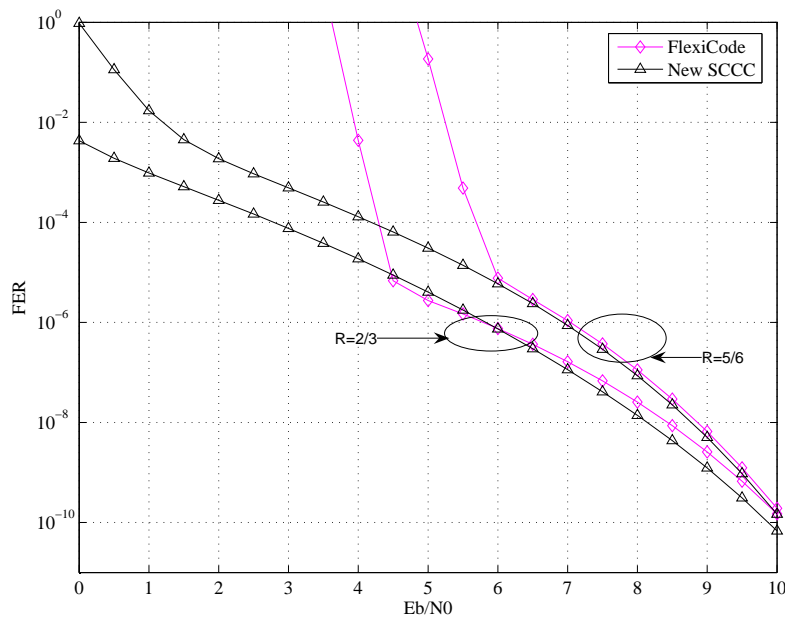

Fig. 5. Upper bounds on $P_{f}(e)$ for the new class of SCCCs (Eq. (3)) and for the "FlexiCode" (Eq. (9)) with $R=2 / 3$ and $R=5 / 6$ with $K=1504$ An AWGN channel is assumed with BPSK modulation.

being $s$ when the input weight is $l$. In particular, referring to Eq. (12), $p_{\mathrm{SPC}}(s \mid l)$ can be also defined as:

$$
p_{\mathrm{SPC}}(s \mid l)=\frac{A_{l, s}^{\mathrm{SPC}}}{\left(\begin{array}{c}
N \\
l
\end{array}\right)}
$$

Its upper bound can be expressed as:

$$
\begin{aligned}
p_{\mathrm{SPC}}(s \mid l) & \leq\left(\frac{s+\delta / 2}{N / J}\right)^{l}\left(\begin{array}{c}
N / J \\
s+\delta / 2
\end{array}\right) \\
& =\left(\frac{s+l}{2 N / J}\right)^{l}\left(\begin{array}{c}
N / J \\
(s+l) / 2
\end{array}\right)
\end{aligned}
$$

The upper bounds on $P_{b}(e)$ for the new class of SCCCs (Eq. (2)) and for the "FlexiCode" (Eq. (8)) are reported in Fig. 4. Fig. 5 reports the upper bounds on $P_{f}(e)$ for the new class of SCCCs (Eq. (3)) and for the "FlexiCode" (Eq. 


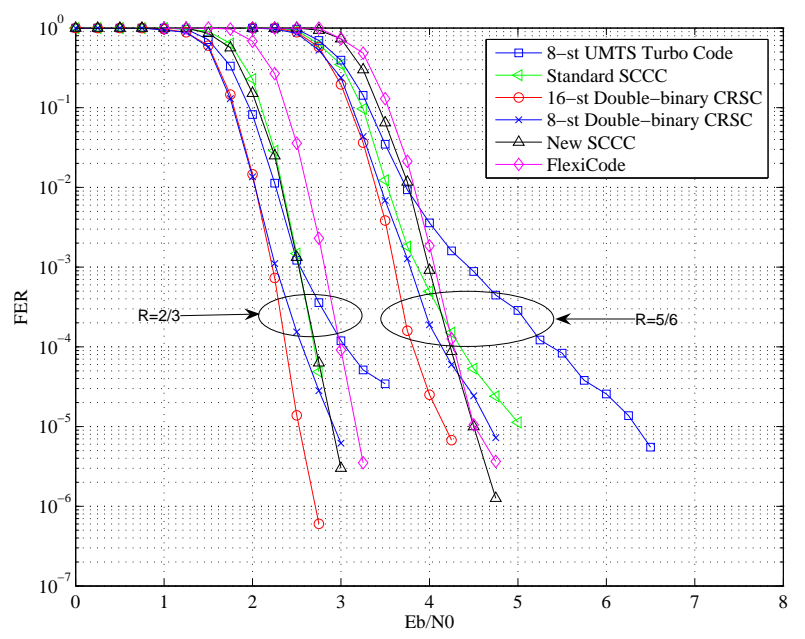

Fig. 6. FER performance after 10 iterations for $R=2 / 3$ and $R=5 / 6$ with $K=1504$. An AWGN channel is assumed with BPSK modulation.

(9)). Two different total code rates are taken for comparison, namely $R=2 / 3$ and $R=5 / 6$. From the figure, both codes show a similar error floor (even if the SCCCs show a slightly better floor) but the new class of SCCCs behaves better in the waterfall region (i.e., for lower SNRs). This, as it will be shown in the following section, leads the new class of SCCCs to present better convergence thresholds w.r.t. the "FlexiCode".

\section{B. Simulation results}

The simulation results are reported in Fig. 6 considering a BPSK modulation scheme on the AWGN channel. In this figure we compare the frame error rate (FER) performances of the codes listed above, w.r.t. the new class of SCCCs proposed in [1-3]. Two different total code rates are taken for comparison, namely $R=2 / 3$ and $R=5 / 6$.

Three different parallel concatenated code structures are considered, namely, the 8-state UMTS Turbo Code (labelled as "8-st UMTS Turbo Code") $[12,13]$, the 8-state Double-Binary Turbo Code (labelled as "8-st Double-Binary CRSC") adopted in the DVB and WiMAX standards $[19,20]$, and the 16-state Double-Binary Turbo Code (labelled as "16-st Double-Binary CRSC") proposed in [14]. The information block length is 188 bytes (1504 bit) [21]. The interleavers for the three PCCCs are optimized as detailed in $[13,14,20]$, whereas for the SCCCs, we use a semi-random interleaver [22]. Ten iterations were simulated and at least 50 erroneous frames were considered for each point in the figure.

When compared to the more complex UMTS Turbo Code (' $\square$ ') and the 8-state Double-Binary Turbo Code (' $\times$ '), the proposed SCCC (' $\triangle$ ') has better performance in the error floor region for both code rates. Whereas both 8-state PCCCs show an error floor for all rates, the proposed SCCC has not still reached the floor at FER $10^{-6}$. The gain is particularly significant for $R=5 / 6$. The improvements are obtained with only a slight loss in convergence threshold: in particular, w.r.t. the UMTS Turbo Code, the loss is not significant.

The error floor experienced by the 8-state PCCCs can be lowered by using 16-states constituents. In particular, the 16-

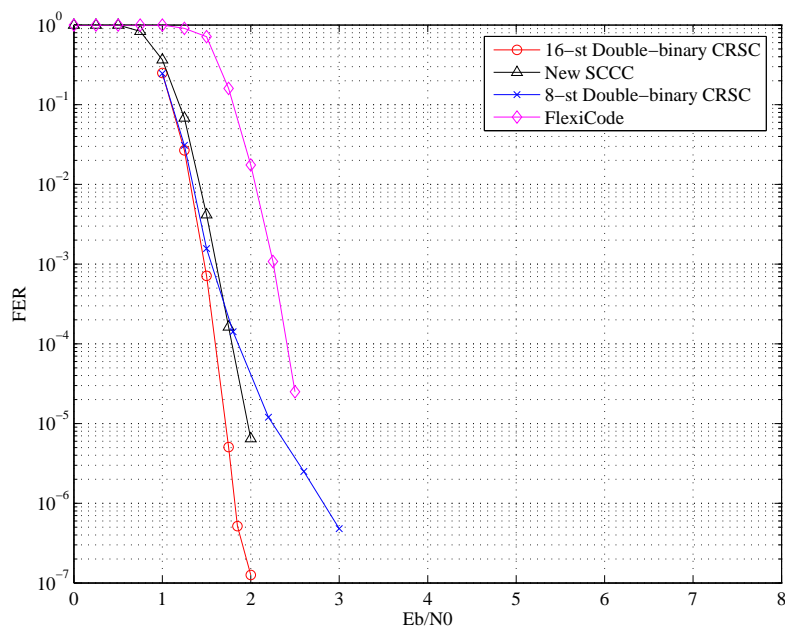

Fig. 7. FER performance after 10 iterations for $R=1 / 2$ with $K=1504$. An AWGN channel is assumed with BPSK modulation.

state DB-PCCC proposed in [14] shows excellent performance in both the WF and the EF regions. For $R=2 / 3$ the proposed SCCC has only a $0.3 \mathrm{~dB}$ loss in convergence threshold w.r.t. the 16-state DB-PCCC, whereas no error floor is observed for the two codes. For $R=5 / 6$ a similar behavior is observed. However, at $R=5 / 6$ the 16 -state DB-PCCC code exhibits an error floor starting at around $6 \times 10^{-6}$, whereas no error floor is observed down to $10^{-6}$ for the proposed SCCC code. Furthermore, it is worth pointing out that the semirandom interleaver used for the proposed SCCC is selected according to [22] with no further attempt for optimization. A careful optimization of the interleaver, as done for the DB-PCCCs, would allow improvements in terms of convergence thresholds and error floor performance. Moreover, in Fig. 6 we also compare the performance of the proposed code with a standard SCCC (' $\triangleleft$ ') of similar complexity and identical semi-random interleaver. The SCCC in Fig. 6 (labelled as "standard SCCC") is constructed by concatenating an 8-states outer code with a 2states inner code [11]. For standard SCCCs, high code rates are obtained by puncturing the outer code, where the puncturing patterns are subsequently optimized for each code rate [23]. Therefore, the code is not rate-compatible. For $R=2 / 3$ the two codes give almost the same performance. However, for higher rates, the standard SCCC encounters an error floor, due to the heavy puncturing of the outer code. In fact, this is one of the main advantages of the proposed structure w.r.t. standard SCCCs: the EF is kept constant and very low for all rates, even with low complexity constituents.

Finally, in Fig. 6 we compare the performance of the proposed code with a new class of serial concatenated codes introduced in [16] and called "FlexiCode". As said above, this class has been selected for comparison since it presents some similarities with the new class of SCCCs described in [1-3]. The similarity is given by the code structure, in the sense that a serial concatenation of an outer and an inner convolutional code, concatenated through an interleaver, is considered, but, in place of using a puncturing device to obtain rate variability, the use of a single parity check (SPC) code is proposed. 


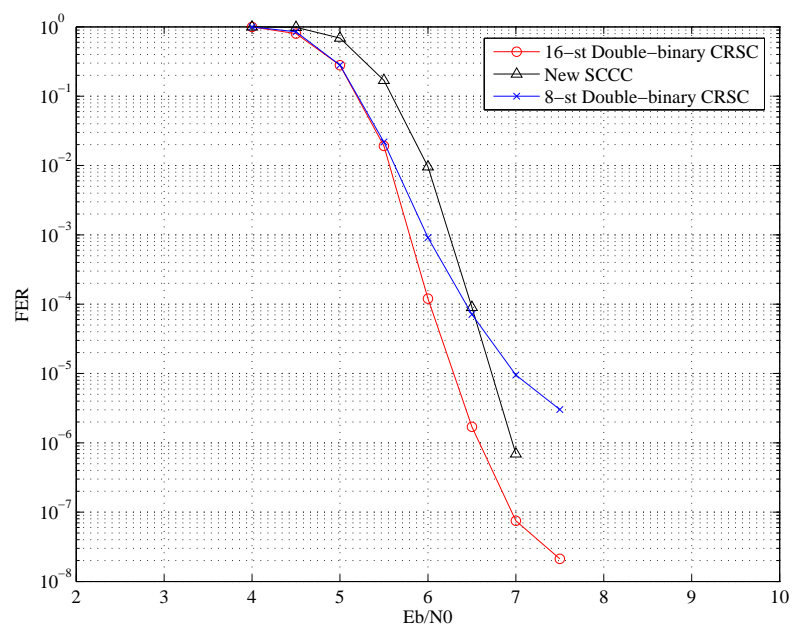

Fig. 8. FER performance after 10 iterations for $R=1 / 2$ with $K=1504$. A Rayleigh fading channel is assumed with 16QAM modulation.

The "FlexiCode" (labelled as "FlexiCode") performance is shown in Figs. 6 and 7 (' $\diamond$ '). In Fig. 7 simulation results are shown for a total code rate $R=1 / 2$. The gain in convergence achieved by the class of SCCCs described in [1$3]$ is significant for a total code rate $R=1 / 2$ : namely, in Fig. 7 a gain in convergence of about $0.7 \mathrm{~dB}$ is shown. This gain decreases with the total code rate, as shown in Fig. 6: in particular, at rate $R=2 / 3$, a gain in convergence of about $0.3 \mathrm{~dB}$ is achieved, whereas at rate $R=5 / 6$ the two classes obtain a comparable convergence threshold, even if the class of SCCCs still gains something in convergence.

As far as complexity is concerned, the number of elementary operations (arithmetic complexity) per decoded bit and memory requirements (memory complexity) is used as complexity measure. The SCCC scheme described in [1-3] is the simplest one in terms of RAM occupation for all block sizes and rates. On the other hand, however, the 8-states and 16-states DoubleBinary schemes only require a small amount of ROM to store the permutation parameters.

The conclusion is that the rate-compatible SCCCs proposed in this paper offer good performance over a broad range of code rates and block lengths, with only limited complexity (4 states constituent codes). 8-states constituent codes have also been analyzed using the proposed structure. The performance is $0.1-0.3 \mathrm{~dB}$ worse in the WF and 1-2 decades better in the EF w.r.t. the proposed 4-state SCCC.

Finally, higher-order M-ary modulation schemes can provide greater spectral efficiency and thus fulfill the data rate requirements of multimedia applications meant for the upcoming DVB standards. To this end, in Figs. 8 and 9 the performance of the SCCC scheme described in [1-3] is compared with the performance of the 8-states and 16-states Double-Binary schemes on a Rayleigh fading channel considering a 16QAM and a QPSK modulation scheme, respectively, and a total rate $R=1 / 2$. Again, as on the AWGN channel with binary modulation, the error floor experienced by the 8-state PCCCs can be lowered by using 16-states constituents. In particular, the 16-state DB-PCCC proposed in [14] is shown also in this

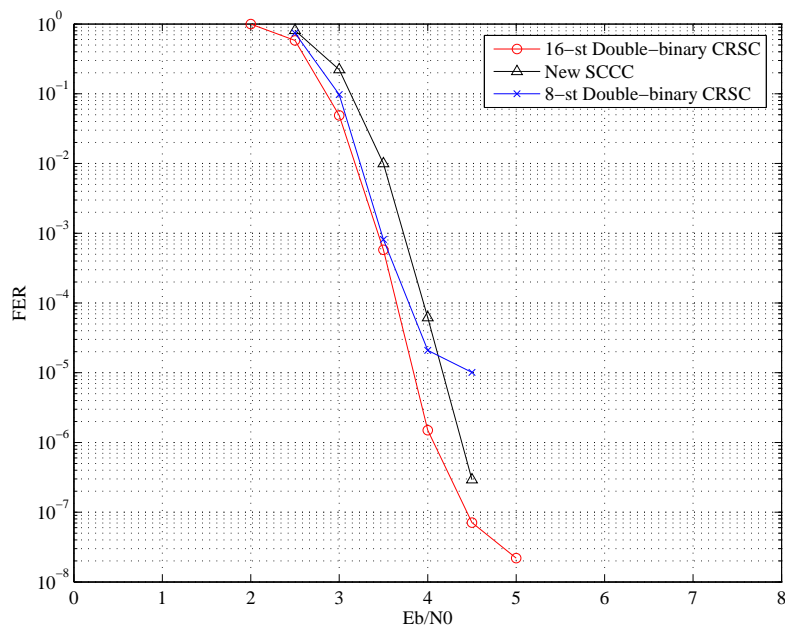

Fig. 9. FER performance after 10 iterations for $R=1 / 2$ with $K=1504$. A Rayleigh fading channel is assumed with QPSK modulation.

case to have excellent performance in both the WF and the EF regions. However, the proposed SCCC scheme is shown to have a loss in convergence up to $0.5 \mathrm{~dB}$ w.r.t. the 16 -state DB-PCCC, but it does not show any error floor, whereas the two PCCCs exhibit an evident error floor for both modulation schemes.

\section{CONCLUSIONS}

In this paper, a number of powerful recent classes of turbo like codes have been analyzed and compared as possible candidates for the upcoming DVB Standards. The final selection has been justified in terms of the best tradeoff between complexity and performance.

Compared to standard rate-dedicated SCCCs, the SCCC codes recently proposed in [1,2] and [3] have a significantly lower error floor for high rates. Compared to more complex PCCCs the proposed codes are competitive in terms of convergence threshold and superior in terms of error floor. Finally, compared to a new class of serial concatenated codes introduced in [16] and called "FlexiCode" the proposed codes exhibit a gain in convergence which is more significant at lower code rates.

\section{REFERENCES}

[1] A. Graell i Amat, G. Montorsi, and F. Vatta, "Analysis and design of rate-compatible Serial Concatenated Convolutional Codes", Proc. of the IEEE 2005 International Symposium on Information Theory, ISIT '05, Adelaide, Australia, September 4-9, 2005, pp. 607-611.

[2] A. Graell i Amat, G. Montorsi, and F. Vatta, "Design and performance analysis of a new class of rate compatible serial concatenated convolutional codes", submitted to the IEEE Transactions on Communications, July 2005, arxiv:cs.IT/0510035, 14 Oct. 2005.

[3] A. Graell i Amat, F. Brännström, and Lars K. Rasmussen, "On the Design of Rate-Compatible Serially Concatenated Convolutional Codes", European Transactions on Communications, vol. 18, no. 5, pp. 519-527, August 2007.

[4] S. Benedetto, R. Garello, G. Montorsi, C. Berrou, C. Douillard, D. Giancristofaro, A. Ginesi, L. Giugno, and M. Luise, "MHOMS: HighSpeed ACM Modem for Satellite Applications", IEEE Wireless Communications, Vol. 12, No. 2, April 2005, pp. 66-77. 
[5] M. Baldi and F. Chiaraluce, "On the Design of Punctured Low Density Parity Check Codes for Variable Rate Systems", Journal of Communication Software and Systems (JCOMSS), Vol. 1, No. 2, December 2005, pp. $88-100$.

[6] M. Baldi, G. Cancellieri, and F. Chiaraluce, "Variable rate LDPC codes for wireless applications", Proc. of the 2006 International Conference on Software, Telecommunications and Computer Networks - SOFTCOM '06, Paper S9-6017-2909, Split, Dubrovnik, Croatia, 29 September - 1 October, 2006.

[7] S. Benedetto and G. Montorsi, "Unveiling turbo codes: some results on parallel concatenated coding schemes", IEEE Trans. on Information Theory, Vol. 42, No. 2, March 1996, pp. 409-428.

[8] I. Land, P. Hoeher, "Partially Systematic Rate 1/2 Turbo Codes", in Proc. Int. Symp. on Turbo Codes and Related Topics, pp. 287-290, Sept. 2000.

[9] F. Babich, G. Montorsi, and F. Vatta, "Some notes on rate-compatible punctured turbo codes (RCPTC) design", IEEE Transactions on Communications, Vol. 52, No. 5, May 2004, pp. 681-684.

[10] S. Benedetto, R. Garello, and G. Montorsi, "A search for good convolutional codes to be used in the construction of turbo codes", IEEE Trans. on Commun., Vol. 46, Sept. 1998, pp. 1101-1105.

[11] S. Benedetto, D. Divsalar, G. Montorsi, F. Pollara, "Serial Concatenation of Interleaved Codes: Performance Analysis, Design, and Iterative Decoding", IEEE Trans. Inf. Theory, Vol. 44, No. 3, May 1998, pp. 909-926.

[12] M. C. Valenti and J. Sun, "The UMTS turbo code and an efficient decoder implementation suitable for software-defined radios", International Journal of Wireless Information Networks, Vol. 8, No. 4, Oct. 2001, pp. 203-215.

[13] "3rd Generation Partnership Project (3GPP)", 3G TS 25.212, v3.5.0, Multiplexing and Channel Coding (FDD), Dec 2000

[14] C. Douillard and C. Berrou, "Turbo codes with rate-m/(m + 1) constituent convolutional codes", IEEE Trans. Commun., Vol. 53, No. 10, Oct. 2005, pp. 1630-1638.

[15] "Interaction channel for satellite distribution systems", DVB, ETSI EN 301790 , vol. 1.2.2, 2000 .

[16] K. M. Chugg, P. Thiennviboon, G. D. Dimou, P. Gray, and J. Melzer "A New Class of Turbo-like Codes with Universally Good Performance and High-Speed Decoding", Proc. of the IEEE Military Communications Conference, MILCOM 2005, Oct. 17-20, 2005, Vol. 5, pp. 3117-3126.

[17] S. Benedetto, and G. Montorsi, Unveiling Turbo Codes: Some Results on Parallel Concatenated Coding Schemes, IEEE Trans. Inf. Theory, vol. 42, pp. 409-429, Mar. 1996.

[18] S. Benedetto, D. Divsalar, G. Montorsi, F. Pollara, "Analysis, Design, and Iterative Decoding Serial Concatenation of Double Serially Concatenated Codes with Interleavers", IEEE Journal on Selected Areas in nCommunications, Vol. 15, No. 2, February 1998, pp. 231-244.

[19] "IEEE standard for local and metropolitan area networks", IEEE $802.16 \mathrm{a}, 2003$

[20] "Interaction channel for satellite distribution systems", DVB, ETSI EN 301790 , Vol. 1.2.2, 2000

[21] "Information technology - Generic coding of moving pictures and associated audio information - Part 1: System", International standard ISO/IEC 13818-1, 1996.

[22] C. Fragouli and R. D. Wesel, "Semi-random interleaver design criteria", Proc. of the IEEE Global Telecommunications Conference - GLOBECOM, Rio de Janeiro, Brazil, Dec. 1999, Vol. 5, pp. 2352-2356.

[23] A. Graell i Amat, G. Montorsi, and S. Benedetto, "Design and decoding of optimal high-rate convolutional codes", IEEE Trans. on Inf. Theory, Vol. 50, No. 5, May 2004, pp. 867-881.
Alexandre Graell i Amat was born in Barcelona, Catalonia, Spain, on January 28th, 1976. He received the M.S. degree in Telecommunications Engineering and the M.S. degree in Electronics Engineeering from Universitat Politcnica de Catalunya, Barcelona, Catalonia, Spain, and the Politecnico di Torino, Torino, Italy, respectively, in 2000, and the Ph.D. degree in Electrical Engineering from the Politecnico di Torino, Torino, Italy, in 2004. From September 2001 to April 2002, he was a Visiting Scholar at the Center for Magnetic Recording Research, University of California at San Diego, La Jolla, CA. From September 2002 to May 2003, he held a visiting appointment at Universitat Pompeu Fabra, and at the Telecommunications Technological Center of Catalonia, both in Barcelona, Catalonia, Spain. During 20012004, he also held a part-time appointment at STMicroelectronics Data Storage Division, Milan, Italy, as consultant on coding for magnetic recording channels. From March 2004 to December 2005, he was a visiting professor at Universitat Pompeu Fabra, Barcelona, Catalonia, Spain. Since January 2006, he has been a PostDoc Researcher at the Department of Electronics at TELECOM Bretagne, Brest, France. His research interests are in the area of communication theory and information theory, and include error-control coding and MIMO systems. Dr. Graell i Amat received the post-doctoral Juan de la Cierva Fellowship of the Spanish Ministry of Education and Science, and the Marie Curie Intra-European Fellowship of the European Commission.

Guido Montorsi was born in Turin, Italy, on January 1, 1965. He received the Laurea in Ingegneria Elettronica in 1990 from Politecnico di Torino, Turin, Italy, with a master thesis, concerning the study and design of coding schemes for HDTV, developed at the RAI Research Center, Turin. In 1992 he spent the year as visiting scholar in the Department of Electrical Engineering at the Rensselaer Polytechnic Institute, Troy, NY. In 1994 he received the $\mathrm{Ph} . \mathrm{D}$. degree in telecommunications from the Dipartimento di Elettronica of Politecnico di Torino. In December 1997 he became assistant professor at the Politecnico di Torino. From July 2001 to July 2002 he spent one year at Sequoia Communications developing baseband algorithm for 3G wireless receivers. In 2003 he became senior member of IEEE and associate professor at Politecnico di Torino. His research interests are in the area of channel coding, particularly on the analysis and design of concatenated coding schemes and study of iterative decoding strategies. Guido Montorsi is author of more the one hundred papers on international journal and conferences.

Francesca Vatta received a Laurea in Ingegneria Elettronica in 1992 from University of Trieste, Italy. From 1993 to 1994 she has been with Iachello S.p.A., Olivetti group, Milano, Italy, as system engineer working on design and implementation of Computer Integrated Building (CIB) architectures. Since 1995 she has been with the Department of Electrical Engineering (DEEI) of the University of Trieste where she received her Ph.D. degree in telecommunications, in 1998, with a Ph.D. thesis concerning the study and design of source-matched channel coding schemes for mobile communications. In November 1999 she became assistant professor at University of Trieste. Starting in 2002, she spent several months as visiting scholar at the University of Notre Dame, Notre Dame, IN, U.S.A., cooperating with the Coding Theory Research Group under the guidance of Prof. D. J. Costello, Jr. Starting in 2005, she spent several months as visiting scholar at the University of Ulm, Germany, cooperating with the Telecommunications and Applied Information Theory Research Group under the guidance of Prof. M. Bossert. She is an author of more than 60 papers published on international journals and conference proceedings. Her current research interests are in the area of channel coding concerning, in particular, the analysis and design of concatenated coding schemes for wireless applications. 